\title{
Literatur
}

1. Bürger, M., Angiopathia diabetica, Georg Thieme. Verlag, Stuttgart (1954). 2. BürGER, M., Altern und Krankheit, VEB Georg Thieme Verlag, Leipzig (1957). - 3. BürGER, M. und H. KNOBLOCH, Münch. med. Wschr. 101, 309 (1959). - 4. HetenYr, G., Acta med. Acad. Sci. Hung. IV, 425 (1963). - Randerath, E. und P. B. Dierzer, III. Kongr. Intern. Diabetes Fed. 1958, S. 54, Georg Thieme Verlag, Stuttgart (1959). - Angervall, L., G. Dotevall und K. LehmanN, Acta med. Scand. 169, 339 (1961). - 7. BencosME, S. A., R. O. West, J. W. KerR und D. L. Wilson, Amer. J. Med. 40, 67 (1966). - 8. Burstern, R., H. T. Blumenthal und S. Soule, Amer. J. Obstetr. Gynec. 74, 96 (1967). - 9. Fuchs, U., Frankf. Zschr. Path. 73, 318 (1964). - 10. Moore, J. M. und I. D. O. Frew, Brit. Med. J. 2, 19 (1965). - 11. Hauss, W. H., Verh. Dtsch. Ges. inn. Med. 69, 554 (1963). - Hauss, W. H., Verh.
Dtsch. Ges. f. Kreislforsch., 30. Tgg., 153 (1964). - 13. Hauss, W. H. und G. JUNGE-HüLSING, Hochdruckforschung II, S. 125, Georg Thieme Verlag, Stuttgart (1965) Symposium Freiburg. 14. JungrHülsing, G., Die Therapiewoche 16, 485 (1966). - 15. Hauss, W. H., G. Junge-Hürsing und H. Themann, Med. Welt 19, 7 (1968). - 16. Otro, H., H. Themann und H. Wagner, Klin. Wschr. 45, 299 (1967). - 17. Hauss, W. H., G. JUNGE-HüLsING und H. Oтто, in: Diabetische Angiopathie S. 229, Akademie Verlag, Berlin (1964): - 18. Schiller, S. und A. Dorfman, Biochim. biophysica Acta (Amsterdam) 16, 304 (1955). - 19. Schiller, S. und A. Dorfman, J. biol. Chemistry 227, 625 (1957). - 20. Glaubitr, D., Verh. Dtsch. Ges. f. Verdauungs- u. Stoffwechselkrankh., 22. Tagg. Wiesbaden 1964. S. 349, S. Karger Verlag, Basel (1965)

\section{Wirkungssynergismus Chondroitin-4-sulfat-Protein abbauender Enzyme des Arteriengewebes}

\author{
Von E. Held, O. Hoefele, G. Reich, U. Stein, E. Werries und E. Buddecke \\ Aus den Pbysiologisch-Chemischen Instituten der Universitäten Tübingen und Münster
}

(Eingegangen am 5. Dezember 1967)

\section{Herrn Prof. Dr. Dr. Ernst Scbütte qum 60. Geburtstag gewidmet}

Im Arteriengewebe des Rindes (Aorta thoracica) sind Hyaluronatglykanohydrolase (EC 3.2.1.35), Chondroitinsulfat-sulfohydrolase (EC 3.1.6.4), N-Acetyl- $\beta$-D-glucosaminidase (EC 3.2.1.30), $\beta$-D-Glucuronidase (EC 3.2.1.31), Kathepsin D (EC 3.4.4.23) und eine saure Carboxypeptidase (EC 3.4.2...) in spezifischen Aktivitäten von 0,01-10 mU/mg Extraktprotein bzw. 0,71-9,4 mU/g Frischgewebe vorhanden und können aus Homogenaten des Frischgewebes 80-1200 fach angereichert werden.

Die Enzyme zeigen bei der Reinigung z. T. gleiches Verhalten und synergistische bzw. successive Wirkung auf Chondroitin-4-sulfatProtein oder dessen Spaltprodukte.

Hyaluronat-glykanohydrolase (Hyaluronidase) und Kathepsin D bauen Kohlenhydrat- bzw. Proteinkomponente des Chondroitin-4-sulfat-Proteins gleichzeitig und ohne gegenseitige Beeinträchtigung ihrer Wirkung ab. Substrat der Arterien-Chondroitin-4-sulfat-sulfohydrolase ist Chondroitin-4-sulfat. Die Abspaltung des Estersulfats durch die Sulfatase wird durch die gleichzeitige Anwesenheit und Wirkung der Hyaluronidase nicht beeinflußt.

Die von der proteolytischen Einwirkung von Kathepsin D auf Chondroitin-4-sulfat-Protein freigesetzten Chondroitin-4-sulfat-Peptide werden durch die saure Carboxypeptidase des Arteriengewebes unter Abspaltung der C-terminalen Aminosäure weiter abgebaut. Der Wirkungssynergismus von Kathepsin D und saurer Carboxypeptidase zeigt sich durch Festlegung ihrer Spaltorte an der oxydierten BKette des Rinderinsulins.

$\beta$-D-Glucuronidase und N-Acetyl- $\beta$-D-glucosaminidase der Aorta lassen sich trotz 1000 facher Reinigung nicht voneinander trennen. In Anwesenheit von $\mathrm{N}$-Acetyl- $\beta$-D-hexosaminidase wird die $\beta$-D-Glucuronidase unter Verschiebung des $\mathrm{pH}-\mathrm{Optimums}$ von $\mathrm{pH} 5,2$ auf $\mathrm{pH} 4,5 \mathrm{pH}$-abhängig aktiviert.

Die Kenntnis der Eigenschaften und Wirkungsweise der Chondroitinsulfat-Protein abbauenden Enzyme erlaubt Rückschlüsse auf den Mechanismus und Abbauweg des Chondroitinsulfats im Arteriengewebe.

Hyaluronate glycanohydrolase (EC 3.2.1.35), chondroitin sulphate sulphohydrolase (EC 3.1.6.4), N-acetyl- $\beta$-D-glucosaminidase (EC 3.2.1.30), $\beta$-D-glucuronidase (EC 3.2.1.31), cathepsin D (EC 3.4.4.23) and an acid carboxypeptidase (EC 3.4.2. ...), with specific activities of $0.01-10 \mathrm{mU} / \mathrm{mg}$ extract protein or $0.71-9.4 \mathrm{mU} / \mathrm{g}$ fresh tissue, are present in bovine arterial tissue (Aorta thoracica) and can be purified $80-1200$ fold from homogenised fresh tissue.

After purification, the enzymes show partly similar activity and synergistic or successive activity towards chondroitin-4-sulphate-protein or its cleavage products.

Hyaluronate glycanohydrolase (hyaluronidase) and cathepsin D simultaneously degrade the carbohydrate and protein components of chondroitin-4-sulphate respectively with no mutual impairment of activity. Chondroitin-4-sulphate is a substrate of arterial chondroitin-4 sulphate sulphohydrolase. Removal of the ester sulphate by the sulphatase is not affected by the presence and simultaneous activity of hyaluronidase.

The chondroitin-4-sulphate-peptides, released by the proteolytic action of cathepsin D on chondroitin-4-sulphate, are further degraded with removal of the C-terminal amino acid by the acid carboxypeptidase of arterial tissue. The mechanism of the synergistic action of cathepsin D and acid carboxypeptidase is shown by locating their sites of cleavage in the oxidised B-chain of bovine insulin.

Despite a 1000 fold purification from the aorta, $\beta$-D-glucuronidase and $N$-acetyl- $\beta$-D-glucosaminidase could not be separated from each other. In the presence of $\mathrm{N}$-Acetyl- $\beta$-D-hexosaminidase, the $\beta$-D-glucutonidase exhibits a $\mathrm{pH}$-dependent activation, 'with a shift of $\mathrm{pH}$ optimum from 5.2 to 4.5 .

From this information on the properties and mode of action of enzymes that degrade chondroitin-sulphate-protein, it is possible to discuss the mechanism and pathway of degradation of chondroitin sulphate in arterial tissue. 
Chondroitinsulfat bzw. seine makromolekularen Proteinverbindungen lassen sich in menschlichen und tierischen Arterien nachweisen (1). Ihre Anreicherung im subintimalen Gewebe und Modellversuche an isoliertem Chondroitin-4-sulfat-Protein sprechen für eine "Molekularsiebfunktion" der Polysaccharidproteine bei der Kontrolle des Stofftransportes in die Arterienwand.

Einbaustudien mit ${ }^{35}$ S-Sulfat zeigen, daß die sulfatierten Polysaccharide des Arteriengewebes einem intensiven Stoffumsatz unterworfen sind (2). Bei konstanter Gewebskonzentration an Chondroitinsulfatprotein müssen Biosynthese und enzymatischer Abbau gleichzeitig und mit gleicher Geschwindigkeit ablaufen. Natur und Wirkungsweise der Chondroitinsulfatprotein abbauenden Enzyme sind nicht nur wegen der engen Beziehungen zwischen Enzymaktivität und Funktion des makromolekularen Chondoitinsulfatproteins, sondern auch wegen möglicher Korrelation kataboler und biosynthetischer Prozesse von Bedeutung.

Die am Abbau des Chondroitinsulfatproteins beteiligten Enzyme des Arteriengewebes lassen sich in löslicher Form gewinnen, voneinander trennen, reinigen und bezüglich ihres Wirkungsmechanismus an natürlichem und synthetischem Substrat untersuchen (3-8). Die Chondroitinsulfatprotein abbauenden Enzyme aus Rinderarteriengewebe, die sowohl die Polysaccharide als auch die Proteinkomponente hydrolytisch spalten, werden im Folgenden als "Glycosaminoglycanohydrolasen" bzw. "Peptidhydrolasen" bezeichnet. Die vorliegende Arbeit faßt unsere Untersuchungen über das funktionelle Zusammenwirken der Glycosaminoglycanohydrolasen und Peptidhydrolasen aus der Rinderaorta zusammen.

\section{Methodik}

Alle angeführten Ergebnisse wurden an Rinderaorten (Arcus aortae und Aorta thoracica) erhalten, die unmittelbar nach Tötung der Tiere entnommen, auf Eis gelegt und nach Abtrennung anhängenden Fett- und Bindegewebes bei $-20^{\circ}$ bis zur Weiterverarbeitung aufbewahrt wurden.

Die Aktivitätsbestimmung der einzelnen Enzyme erfolgt jeweils unter optimalen Bedingungen, die Darstellung der gereinigten Enzympräparate wurde nach früher beschriebenen Methoden durchgefübrt.

Reinigung und Aktivitätsbestimmung der Enzyme: N-Acetyl$\beta$ D-glucosaminidase( EC 3.2.1.30) mit Phenyl- $\beta$-D-N-acetylglucosaminid als Substrat (3), Hyaluronat-glycanohydrolase (EC 3.2.1.35) mit Kalium-Chondroitin-4-sulfat als Substrat (4),
$\beta$-D-Glucuronidase (EC 3.2.1.31) mit Phenolphthalein- $\beta$-D-Glucuronid und dem aus Kalium-Chondroitin-4-sulfat durch erschöpfenden Abbau mit Testeshyaluronidase (EC 3.2.1.35) erhaltenen Tetrasaccharid GlcUA-GalNAc-4-sulfat und GlcUAGalNAc-4-sulfat ${ }^{1}$ ) als Substrate (5), saure Carboxypeptidase (EC 3.4.2. ...) mit synthetischem L-Ser-L-Leu-L-Leu, der oxydierten B-Kette des Insulins und dem durch Kathepsin $\mathrm{D}$ abgebauten Chondroitin-4-sulfatprotein als Substrate (6), Chondroitinsulfat-Sulfohydrolase (EC 3.1.6.4) $\mathrm{mit}{ }^{35} \mathrm{~S}$-Chondroitin-4-sulfat als Substrat (7), Protease vom Kathepsin D-Typ (EC 3.4.4.23) mit denaturiertem Hämoglobin, der oxydierten B-Kette des Insulins und Chondroitin-4-sulfatprotein als Substrat (8). Alle Substrate wurden durch chemische Sythese oder durch Isolierung aus Rinderorganen, Chondroitin-4-sulfatprotein aus Rindernasenknorpelfrischgewebe (9), dargestellt.

\section{Ergebnisse}

Eine Übersicht über die bisher aus Arteriengewebe isolierten, Chondroitinsulfatprotein abbauenden Enzyme und ihre Eigenschaften gibt Tabelle 1. Die in der Tabelle aufgetührten Enzyme weisen insofern gemeinsame Merkmale und funktionelle Eigenschaften auf, als sie 1. alle in einem $\mathrm{pH}$-Bereich $z$ wischen 4 und 5 optimal wirksam sind und 2. alle Chondroitin-4sulfatprotein oder dessen Spaltprodukte als Substrate angreifen können. Die experimentellen Ergebnisse, die für einen Wirkungssynergismus der Glycosaminoglycanohydrolasen sprechen, sind in den Abschnitten 1 bis 4 aufgeführt. Eine synergistische bzw. successive Wirkung zweier Enzyme wird angenommen, wenn a) beiden Enzyme sich über mehrere Reinigungsschritte gemeinsam anreichern lassen und dabei keine oder keine vollständige Trennung erfolgt und wenn $b$ ) außerdem beide Enzyme das gleiche Substrat gleichzeitig und unabhängig voneinander oder in der Weise umsetzen, daß die Einwirkung des einen Enzyms Voraussetzung für die Wirkung des zweiten Enzyms ist. Eine Wechselwirkung $z$ wischen $z$ wei Enzymen wird ferner angenommen, wenn sich eine Aktivierung eines Enzyms durch ein zweites Enzym nachweisen läßt.

1. Gemeinsame Anreicherung und gleichzeitige Wirkung von Hyaluronat-glycanohydrolase (Hyaluronidase) und Kathepsin D aus Rinderaorta auf Chondroitin-4-sulfat-Protein.

Homogenate von Rindergewebe in 1proz. Natriumchloridlösung enthalten nach 2 Frier- $\left(-20^{\circ}\right)$ - Tau $\left(0^{\circ}\right)$ - Schritten und Citratfällung (Dialyse gegen

1) Abkïrzungen: GalNAc $=$ N-Acetyl-D-Galaktosaminrest GlcUA $=\beta$-D-Glucuronsäurerest

Tab. 1

Glycosaminoglycanohydrolasen und Peptidhydrolasen aus Arteriengewebe (Aorta, Rind)

\begin{tabular}{|c|c|c|c|c|}
\hline Enzym & EC & Natürliches Substrat & pH-Optimum & Anreicherung \\
\hline Protease (Kathepsin D) & 3.4.4.23 & $\begin{array}{l}\text { Proteinkomponente des Chondroitinsulfatprotein } \\
\text { (u. a. Proteine) }\end{array}$ & 4,0 & 980 \\
\hline Saure Carboxypeptidase & $3.4 .2 . \ldots$ & Oligopeptide mit C-terminalen Leu, Val, Phe, Tyr & 4,6 & 225 \\
\hline Hyaluronat-Glycanohydrolase & 3.2.1.35 & Hyaluronat, Chondroitin 4- und 6-sulfat, Chondroitin & 4,1 & 440 \\
\hline Chondroitinsulfat-sulfohydrolase & 3.1.6.4 & Chondroitin 4-sulfat & 5,0 & 85 \\
\hline$\beta$-D-Glucuronidase & 3.2.1.31 & $\begin{array}{l}\text { Tetrasaccharide und höhere geradzahlige Oli- } \\
\text { gosaccharide des Hyaluronat und Chondroitinsulfat }\end{array}$ & $\begin{array}{c}5,2 \\
(4,5)\end{array}$ & 1200 \\
\hline N-Acetyl- $\beta-D$-glucosaminidase & 3.2.1.30 & $\begin{array}{l}\text { Trisaccharide und höher ungeradzahlige Oli- } \\
\text { gosaccharide des Hyaluronat }\end{array}$ & 4,5 & 600 \\
\hline
\end{tabular}




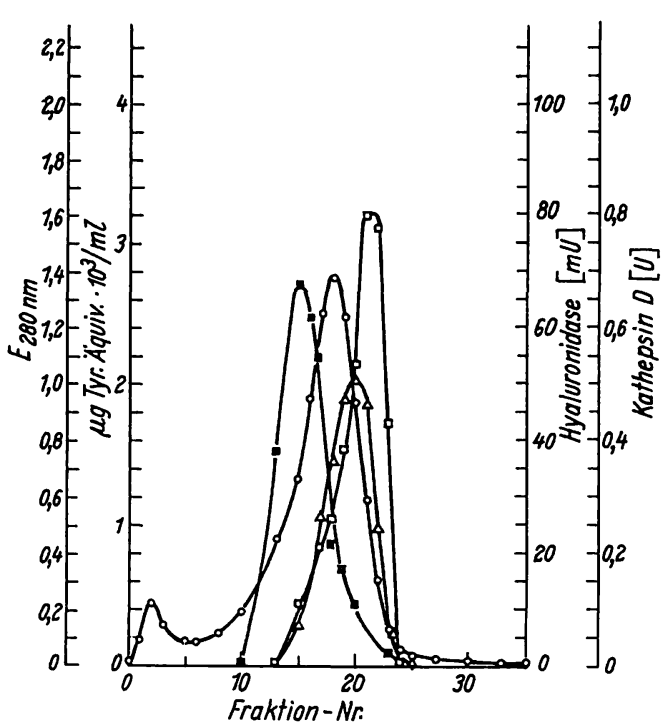

Abb. 1

Elutionsdiagramm von $50 \mathrm{mg}$ Enzymprotein (spezifische Proteaseaktivität $260 \mathrm{mU}$ ) nach Gelfiltration an Sephadex G $100(0,05 \mathrm{M} \mathrm{Ci}$ trat-Phosphatpuffer $\mathrm{pH} 4,0+0,1 \mathrm{M} \mathrm{NaCl}$ )

linke Ordinate: Proteingehalt (E $280 \mathrm{~nm}) \circ-0$ linke Ordinate: Kathepsin D-Aktivität in $\mu \mathrm{g}$ Tyrosinäquivalenten pro $\mathrm{m} l$ und $15 \mathrm{Min} . \Delta-\Delta$ rechte Ordinate: Spezifische Kathepsin D-Aktivitłä rechte Ordinate: $\begin{gathered}\text { in } U \text { ezifische Aktivität der Hyalur- } \\ \text { onidase }\end{gathered}$

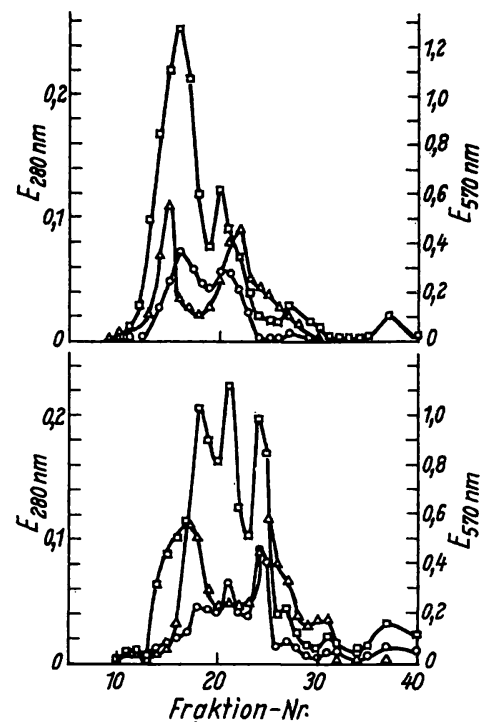

Abb. 2

Elutionsdiagramm der dialysablen Peptidspaltprodukte nach Kathepsin $D$-Abbau von nativem Chondroitin4-sulfatprotein (obere $\mathrm{Abb}$.) und von mit Hyaluronat-Glycanohydrolase teilweise abgebautem Chondroitin-4. sulfatprotein (untere Abb.). Gelfiltration an Sephadex $\mathrm{G} 25$ in $0,5 \mathrm{M} \mathrm{NaCl}$ linke Ordinate: $\mathrm{E} 280 \mathrm{~nm}$ 。rechte Ordinate: Ninhydrinreaktion (E $570 \mathrm{~nm}) \Delta-\Delta$

rechte Ordinate: Ninhydrinreaktion

(E $570 \mathrm{~nm}$ ) nach alkalischer Hydrolyse

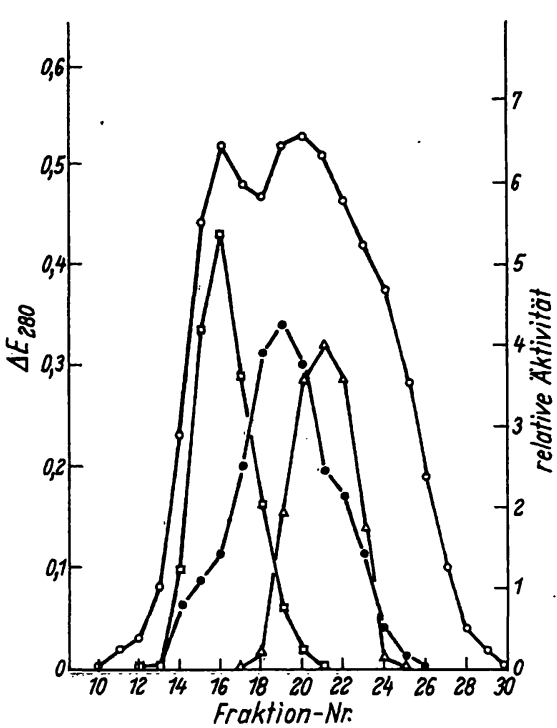

Abb. 3

Elutionsdiagramm von $75 \mathrm{mg}$ Enzymprotein (Acetatfüllung pH 4,6 nach 1. c. 6) nach Gelfiltration an Sephadex G 100

linke Ordinate: E $280 \mathrm{~nm}$ orechte Ordinate: relative Aktivität, Arylsulfat-Sulfohydrolase, $\square-\square$ Chondroitin-4-sulfat-sulfohydrolase --. Hyaluronat-Glykanohydrolase $\Delta-\Delta$
0,05M Citrat-Puffer pH 3,5) eine spezifische Kathepsinaktivität von $1,26 \mathrm{mU} / \mathrm{mg}$ Protein und eine spezifische Hyaluronidaseaktivität von $0,07 \mathrm{mU} / \mathrm{mg}$ Protein. Wird der salzfreie lyophilisierte Úberstand 4 säulenchromatographischen Reinigungsschritten unterworfen (DEAECellulose, TEAE-Cellulose, Sephadex-G 75 und G 100), so läßt sich die spezifische Kathepsinaktivität auf $560 \mathrm{mU} / \mathrm{mg}$ Protein, die spezifische Aktivität der Hyaluronidase auf $67 \mathrm{mU} / \mathrm{mg}$ Protein steigern. Dies entspricht einer Anreicherung gegenüber dem Homogenat auf das $440 \mathrm{bzw}$. 880fache. Abbildung 1 zeigt das Ergebnis der Gelfiltration an Sephadex G 100 des durch Austauschchromatographie und Gelfiltration an G 75 vorgereinigten Enzympräparates, das Kathepsin und Hyaluronidase getrennt, jedoch im gleichen Proteingipfel enthält. Trotz mehrhundertfacher Reinigung verhalten sich beide Enzymproteine ähnlich, bemerkenswert ist dabei, daß die Aktivität der Hyaluronidase weder durch die anwesende Protease selbst, noch durch den zunehmenden Abbau der Proteinkomponente des Chondroitinsulfatproteins beeinflußt wird. Denn die Hyaluronidase setzt Chondroitin-4-sulfatprotein und das proteinfreie Chondroitin-4-sulfat mit gleicher Geschwindigkeit um und weist für beide Substrate die gleiche Michaeliskonstante $(1,25 \mathrm{~mm})$ auf.

Umgekehrt ist die Spaltbarkeit der Proteinkomponente des Chondroitin-4-sulfatproteins durch das Kathepsin nur geringfügig von dessen Kohlenhydratgehalt $a b-$ hängig: Denn Chondroitin-4-sulfatprotein und ein
Chondroitin-4-sulfatprotein-Präparat, dessen Kohlenhydratgehalt durch Abbau mit Testeshyaluronidase zuvor zu $75 \%$ entfernt worden war, wurden unter vergleichbaren Bedingungen (gleicher Proteingehalt des Inkubationsansatzes) durch Aortenkathepsin in etwa gleichem Ausmaß abgebaut. Die Gesamtmenge der niedermolekularen (dialysablen) Spaltprodukte lag gemessen an der nach alkalischer Hydrolyse mit der Ninhydrinreaktion bestimmten Menge der Aminosäuren - für das Kohlenhydrat abgebaute Substrat nur um $12 \%$ höher als für das native Chondroitinsulfatprotein. Abbildung 2 zeigt das Elutionsdiagramm der während der Proteolyse gebildeten durch Unterdruckdialyse eingeengten und durch Gelfiltration fraktionierten Spaltprodukte, die beim Abbau von nativem Chondroitin-4-sulfatprotein (oberer Teil der Abbildung) und hyaluronidasevorbehandeltem Chondroitin-4-sulfatprotein (unterer Teil) erhalten wurden. Es ist demnach anzunehmen, daß Hyaluronidase und Kathepsin in der Arterienwand gleichzeitig und ohne gegenseitige Beeinträchtigung die Kohlenhydrat- und Proteinkomponente des Chondroitin-4-sulfatprotein abbauen können.

2. Gleichzeitige und unabhängige Wirkung von Chondroitin-4-sulfatsulfohydrolase (Chondroitinsulfatase) und Hyaluronat-glycanohydrolase (Hyaluronidase) auf Chondroitin-4-sulfat.

Beweise für die Existenz eines am Abbau des Chondroitin-4-sulfat beteiligten sulfatesterspaltenden En- 
zyms im Arteriengewebe konnten durch Isolierung einer Chondroitinsulfat-sulfohydrolase erbracht werden. Das Enzym ist nicht mit der Arylsulfatase (EC 3.1.6.1) identisch. $\mathrm{Da}$ das aus Rinderarterien 85-fach angereicherte Enzym auf das intakte Chondroitinsulfat wirkt (pH-Optimum 4,6), jedoch auch Hyaluronidase Chondroitinsulfat ohne vorherige Desulfatierung zu geradzahligen sulfatierten Oligosacchariden abbauen kann (pH-Optimum 4,1), können beide Enzyme in der Initialphase des Chondroitinsulfat-Abbaues das gleiche Substrat (C-4-S) gleichzeitig umsetzen. Auch bei der Anreicherung der Chondroitin-4-sulfat-sulfatase nimmt die Aktivität der Hyaluronidase zunächst zu. Erst nach 40facher Reinigung der Chondroitin-sulfohydrolase (bezogen auf das Homogenat) wird die in den gleichen Reinigungsschritten (Ammonsulfatfällung $\mathrm{pH}$ 4,6) 20fach angereicherte Hyaluronidase durch Gelfiltration abgetrennt.

Die vorherige Einwirkung von Chondroitinsulfatase begünstigt den nachfolgenden Abbau durch die Hyaluronidase, da nach Entfernung des Sulfats die Reaktionsgeschwindigkeit für die glykosidische Spaltung der Polysaccharidkette zunimmt. Im Gegensatz hierzu hat die Hyaluronidase jedoch keinen Einfluß auf die enzymatische Desulfatierung des intakten Chondroilin-4sulfat durch die Chondroitinsulfatase. Dies zeigt sich darin, daß die Aktivität der Chondroitinsulfatase nach Abtrennung der Hyaluronidase konstant bleibt bzw. daß bei überlappenden Aktivitäten von Chondroitinsulfatase und Hyaluronidase während der säulenchromatographischen Reinigungsschritte im Überschneidungsbereich beider Enzymaktivitäten keine Steigerung der Chondroitinsulfataseaktivität durch die gleichzeitig anwesende Hyaluronidase eintritt (Abb. 3).

3. Wirkungssynergismus von Kathepsin D (Protease) und saurer Carboxypeptidase.

Die in der Rinderaorta vorhandene nachweisbare proteolytische Aktivität entspricht einer Protease vom Typ des Kathepsins $D$, das neben anderen Proteinsubstraten auch hochmolekulare ChondroitinsulfatProteine angreift (8). Bei Einwirkung von $100 \mu \mathrm{g}$ Aortenkathepsin (spezifische Aktivität 1,2 mU/mg Protein) sinkt die reduzierte Viskosität einer Lösung von $5 \mathrm{mg}$ Chondroitinsulfat-Protein $/ \mathrm{ml}$ innerhalb weniger Minuten auf die Hälfte des Ausgangswertes ( $\eta$ red.
2,8) ab. Die Ninhydrinreaktion erfährt dabei eine entsprechende Zunahme. Die Wirkung des Kathepsins auf das Chondroitinsulfat-Protein wird durch die Aktivität eines zweiten, Peptidbindungen spaltenden Arterienenzyms, der sauren Carboxypeptidase, ergänzt. Die saure Carboxypeptidase, die von Peptiden Cterminale Aminosäuren hydrolytisch abspaltet (7), ist allein an Chondroitsulfat-Proteinen nicht wirksam. Bei Einwirkung auf die durch Kathepsin D freigesetzten proteolytischen Spaltprodukte des Chondroitinsulfat-Proteins lassen sich jedoch freies Leucin, Valin, Phenylalanin und Tyrosin nachweisen. Das Wirksamwerden der sauren Carboxypeptidase wird also erst durch die vorherige Tätigkeit des Kathepsins ermöglicht, das proteolytische Spaltprodukte des Chondroitin-4-sulfatProtein (Chondroitin-4-sulfat-Peptide) mit für die saure Carboxypeptidase angreifbaren C-terminalen Aminosäureresten liefert. Die successive Wirkung beider Enzyme läßt sich durch Untersuchung ihrer Spaltorte an der B-Kette des Insulins lokalisieren. Nach hydrolytischer Öffnung von vier Peptidbindungen an den Positionen 15/16, 16/17, 24/25 und 25/26 durch das Kathepsin $\mathrm{D}$ vermag die saure Carboxypeptidase die terminalen Aminosäuren Phenylalanin in Position 24 und Leucin in Position 15 abzuspalten (Abb. 4). Die Spaltung weiterer Peptidbindungen durch Kathepsin D und saure Carboxypeptidase ist wahrscheinlich, erfolgt jedoch unter geringerer Reaktionsgeschwindigkeit.

4. Wechselwirkungen $z$ wischen $\beta$-D-Glucuronidase und N-Acetyl- $\beta$-D-glucosaminidase.

Beim Abbau der Chondroitinsulfatkomponente wird der Sulfatester von Chondroitin-4-sulfat durch die Chondroitinsulfatase desulfatiert und durch die Hyaluronidase zu geradzahligen Oligosacchariden depolymerisiert. Die entstehenden Oligosaccharide werden dann durch die alternierende Wirkung. von $\beta$-D-Glucuronidase und $\mathrm{N}$-Acetyl- $\beta$-D-glucosaminidase weiter zu Monosacchariden abgebaut. Die engen funktionellen Wechselwirkungen $z$ wischen $\beta$-D-Glucuronidase und $N$-Acetyl- $\beta$-D-glucosaminidase zeigen sich darin, daß sich beide Enzyme über 8 verschiedene $z$. T. säulenchromatographische Trennungsverfahren $600 \mathrm{bzw}$. 1200 fach anreichern lassen und auf der Endstufe der Reinigung im Elutionsdiagramm (Abb. 5) in identischer Position erscheinen.

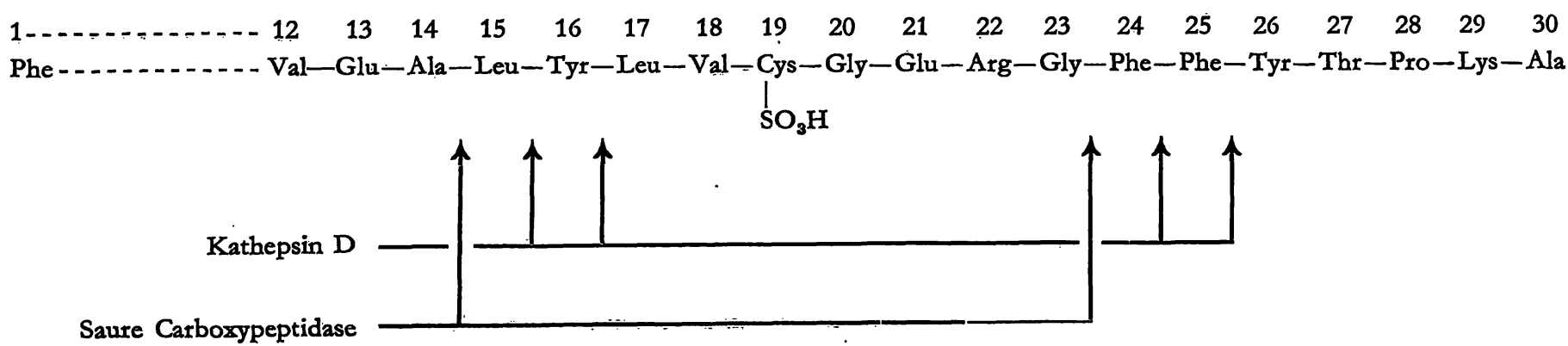

Abb. 4

Wirkungsorte des Kathepsin D und der sauren Carboxypeptidase aus Arteriengewebe an der B-Kette des Insulins 


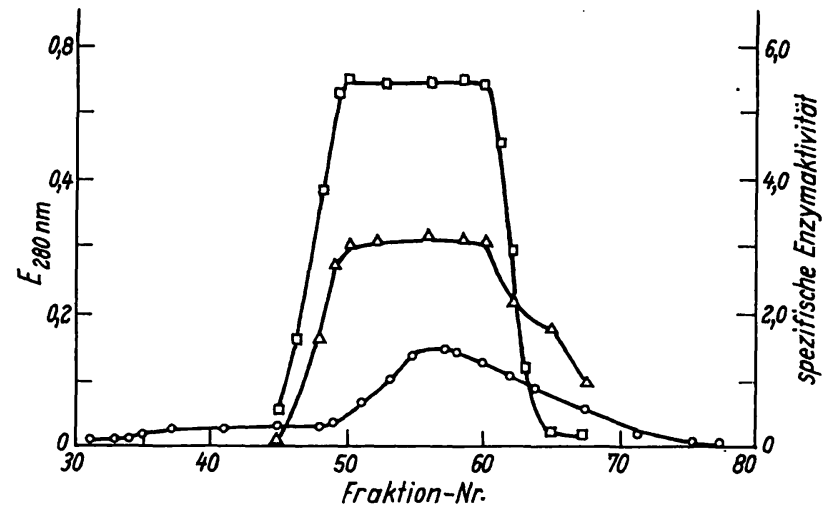

Abb. 5

Elutionsdiagramm eines $\beta$-D-Glucuronidase und $\mathrm{N}$-Acetyl- $\beta$-DGlucosaminidase enthaltenden Enzympräparates $(7 \mathrm{mg}$ in $0,1 \mathrm{M}$ Citratpuffer $\mathrm{pH} 5,2)$ nach Chromatographie an Sephadex G 200 (Säulenvolumen $1,5 \times 300 \mathrm{~cm}$ ). Endstufe der Reinigung mit 1200 facher Anreicherung der $\beta$-D-Glucuronidase

linke Ordinate: Proteingehalt (E $280 \mathrm{~nm}) \circ$

rechte Ordinate: spezifische Aktivitäten der $\beta$-D-Glucuronidase $\times 10^{-1}(\square-\square)$ und der $N$-Acetyl- $\beta$-D-glucosaminidase $(\Delta-\Delta)$

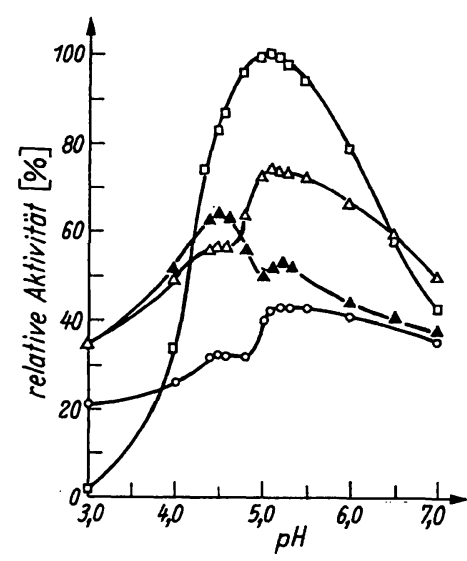

Abb. 6

$\mathrm{pH}$-abhängige Aktivierung der $\beta$ - $\mathrm{D}$-Glucuronidase aus Rinderarteriengewebe durch Glucuronidase-freie $N$-Acetyl $\beta$-D-glucosaminidase

$\Longrightarrow$ nichtaktivierte $\beta$-D-Glucuronidase (enthaltend $0,2 \mathrm{mU}$ $\beta$-D-Glucuronidetyl- $\beta$-D-glucosaminidase)

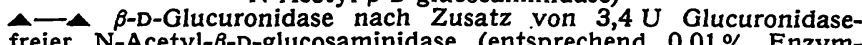
freier N-Acetyl- $\beta$-D-glucosaminidase (entsprechend $0,01 \%$ Enzym$\Delta-\Delta \quad B$-D-Glucuronidase nach Zusatz von $0,01 \%$ Rinderserum$\square$ - $\beta$-D-Glucuronidase nach Zusatz von $0,03 \%$ Desoxyribonucleinsäure

Darüber hinaus hat die N-Acetyl- $\beta$-D-glucosaminidase die Fähigkeit, die $\beta$-D-Glucuronidase in spezifischer, $\mathrm{pH}$-abhängiger Weise zu aktivieren. Die Aktivitätskurve gereinigter $\beta$-D-Glucuronidase zeigt in $0,1 \mathrm{M}$ Acetatpuffer $\mathrm{pH}-\mathrm{Optima}$ bei 5,2 und $\mathrm{pH} 4,5$, wobei das Verhältnis der Glucuronidaseaktivitäten bei $\mathrm{pH} 5,2$ und $\mathrm{pH}$ 4,5 1,3:1 beträgt. Bei Zusatz von kristallisiertem Rinderserumalbumin (Endkonzentration 0,03\%) wird der bekannte über den gesamten $\mathrm{pH}$-Bereich gleichmäßige Aktivierungseffekt sichtbar, wobei jedoch das Verhältnis der Glucuronidase-Aktivitäten bei pH 4,5 und 5,2 konstant bleibt. Bei Zusatz von glucuronidasefreier N-Acetyl- $\beta$-D-glucosaminidase (die durch Lyophilisieren hochgereinigter Enzympräparate mit $\beta$-DGlucuronidase und N-Acetyl- $\beta$-D-glucosaminidaseaktivi- täten gewonnen wird, wobei die $\beta$-D-Glucuronidase inaktiviert wird) erfolgt jedoch eine Aktivierung der $\beta$-D-Glucuronidase, die bei $\mathrm{pH} 4,5$ stärker ausgeprägt ist, als bei pH 5,2 (Abb. 5). Dadurch verschiebt sich das Maximum der $\beta$-Glucuronidaseaktivität von $\mathrm{pH} 5,2$ auf $\mathrm{pH} 4,5$ und fällt damit mit dem Aktivitätsmaximum der $\mathrm{N}$-Acetyl- $\boldsymbol{\beta}$-D-glucosaminidase zusammen.

Der Aktivierungseffekt ist abhängig von der Menge der zugesetzten $\mathrm{N}$-Acetyl- $\boldsymbol{\beta}$-D-glucosaminidase. Die Werte der Abbildung 6 lassen erkennen, daß nach Hexosaminidasezusatz die Glucuronidaseaktivität bei $\mathrm{pH}$ 4,5 um $133 \%$, bei $\mathrm{pH} 5,2$ jedoch nur um $24 \%$ ansteigt. Die $\mathrm{pH}$-abhängige Aktivierung $\operatorname{der} \beta$ - $\mathrm{D}$-Glucuronidase durch die $N$-Acetyl- $\beta$-D-glucosaminidase weist auf enge Beziehung $z$ wischen beiden Enzymen hin.

\section{Diskussion}

Die simultane bzw. successive Wirkung von sechs hydrolytischen Enzymen beim Abbau des Chondroitinsulfat-Proteins des Arteriengewebes läßt sich durch zahlreiche experimentelle Hinweise belegen. Zwar kann ein kontinuierlicher enzymatischer Abbau des Chondroitinsulfat-Proteins auch aus der Tatsache des im Arteriengewebe nachweișbaren ständigen Stoffumsatzes des Chondroitinsulfat-P1oteins geschlossen werden, doch war eine Formulierung des Mechanismus und des Abbauweges im Rahmen des katabolen Stoffwechsels erst nach Kenntnis der Eigenschaften und Wirkungsweise der beteiligten Enzyme möglich. So läßt sich z. B. aus den beim Studium der Hyaluronidase und Chondroitinsulfatase gewonnenen Ergebnisse ableiten, daß es sich bei der Wirkung dieser beiden Enżyme nicht um eine Konkurrenz um das gleiche Substrat, sondern um den gleichzeitigen enzymatischen Abbau eines Makromoleküls durch zwei verschiedene Enzyme handelt und daß ferner in diesem Falle das Wirksamwerden des einen Enzyms nicht die vorangehende oder gleichzeitige Wirkung des anderen Enzyms zur Voraussetzung hat. Ein zusammenfassendes Schema des Abbaues von Chondroitin-4-sulfat gibt Abbildung 8.

Dem Synergismus der Chondroitinsulfat-Protein abbauenden Enzyme (Glykosaminoglykanohydrolasen) entspricht ein analoges Verhalten bei ihrer Reindarstellung. So lassen sich . Hyaluronidase, Sulfatase, Glucuronidase, Hexosaminidase und Carboxypeptidase nicht nur durch fraktionierte Ammoniumsulfatfällung (Ammoniumsulfatkonzentration von 15-25 Gew-\%) gemeinsam anreichern, sondern auch bei den säulenchromatographischen Reinigungssschritten erfolgt eine Trennung der einzelnen Enzymproteine nur unvollständig (Hyaluronidase und Protease) oder überhaupt nicht (Hexosaminidase und Glucuronidase). $\mathrm{DaB}$ es sich bei den Enzymen Glucuronidase und Hexosaminidase um einen nur langsam disssoziierenden Bienzymkomplex handelt, ist $z$ war mit dem Elutionsdiagramm vereinbar, aber nicht bewiesen. Auf jeden Fall deutet das Phänomen, daß die $\beta$-Hexosaminidase das zweite 
$\mathrm{pH}-$ Optimum der $\beta$-Glucuronidase zu induzieren bzw. zu verstärken vermag auf eine enge Wechselwirkung (kooperative allosterische Aktivierung) beider Enzyme hin.

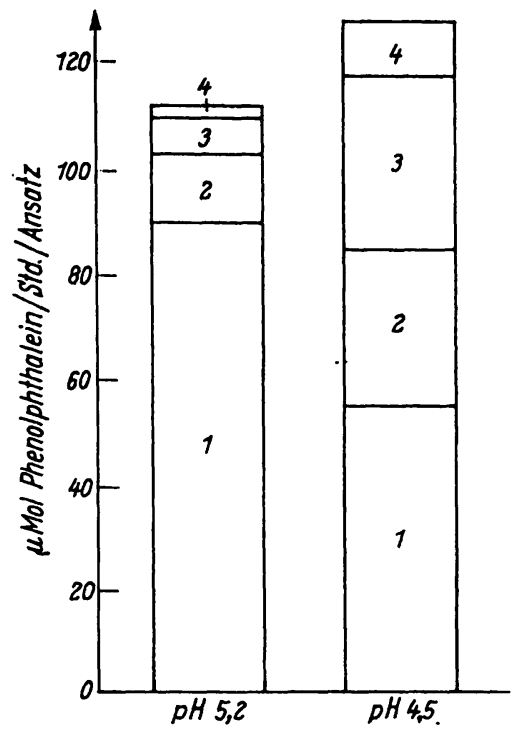

Abb. 7

Aktivierung der $\beta$-D-Glucuronidase bei $\mathrm{pH} 5,2$ und $\mathrm{pH} 4,5$ in Abhängigkeit von der Konzentration der zugesetzten N-Acetyl$\beta$-D-glucosaminidase

$1=$ nichtaktivierte $\beta$-D-Glucuronidase (enthaltend $0,29 \mathrm{mU} \mathrm{N}$ Acetyl- $\beta$-D-glucosaminidase). 2,3 und $4=$ Aktivität nach Zusatz steigender Mengen $\mathrm{N}$-Acetyl- $\beta$-D-glucosaminidase $(\beta$-D-glucuronidasefrei). $2=27 \mathrm{mU}(=8 \mu \mathrm{g}$ Protein), $3=82 \mathrm{mU}(=24 \mu \mathrm{g}$ Protein), $4=8 \mathrm{~g}$ Protein $), 3=82 \mathrm{mU}(=40 \mu \mathrm{g}$ Protein $)$

Chondroitin-4-sulfat

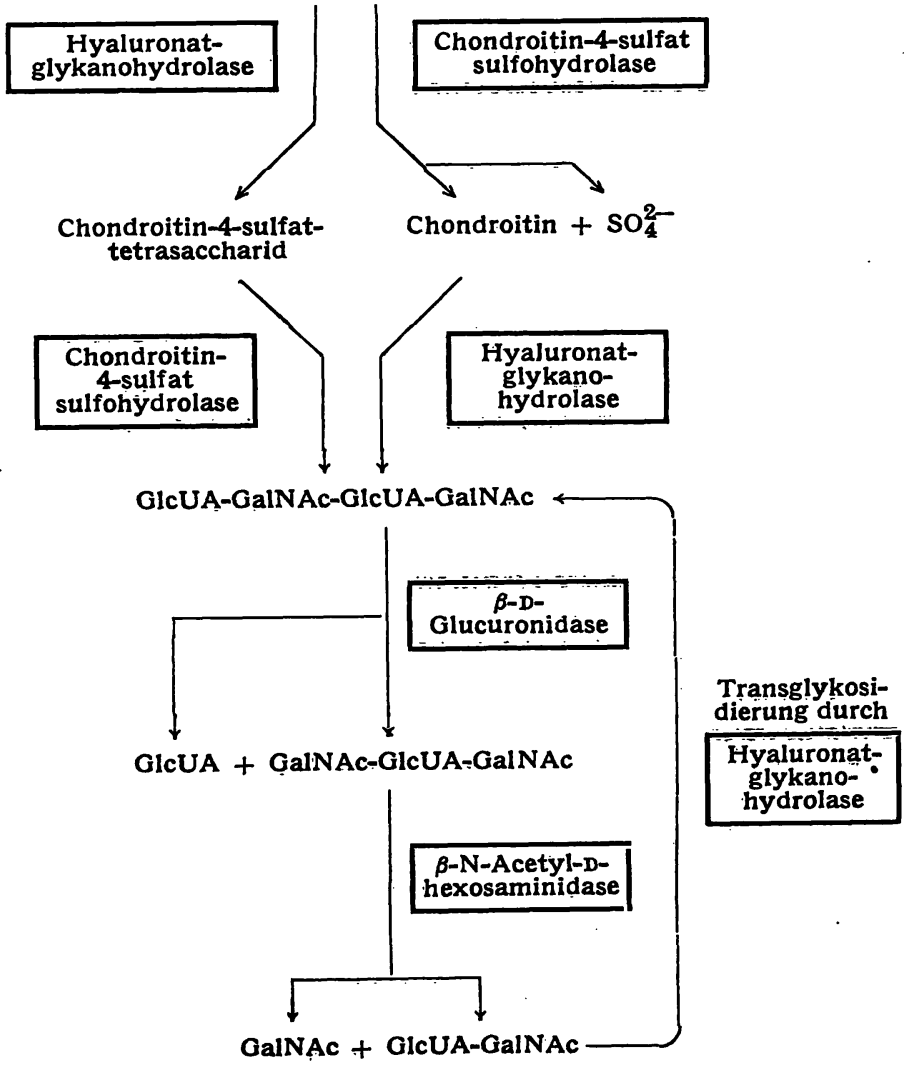

Abb. 8

Schema des Abbaus von Chondroitin-4-sulfat durch die Glykosaminoglykanohydrolasen des Arteriengewebes
Wird Chondroitinsulfat-Protein einem vollständigen hydrolytischen Abbau durch die beschriebenen Enzyme unterworfen, so treten als Endprodukte Glucuronsäure, N-Acetyl-galaktosamin, anorganisches Sulfat (so wie das hier nicht berücksichtigte Chondroitinsulfatkette und Proteinanteil verknüpfende O-Seryl-Trisaccharid (Gal-Gal-Xyl-Ser) oder dessen Spaltprodukte) auf. Für den Abbau des Proteinanteils des Chondroitinsulfat-Protein muß neben der Aktion des Kathepsin D und der Carboxypeptidase das Eingreifen weiterer peptidspaltender Enzyme (wie z. B. der Leucinaminopeptidase (EC 3.4.1.1) (6,11) des Arteriengewebes, deren Wirkung sich jedoch im Gegensatz zu Kathepsin und Carboxypeptidase auf den Neutralbereich beschränkt, gefordert werden.

Bei einer speziellen Diskussion des Abbaus der Chondroitinsulfatkomponente läßt sich aufgrund der Kenntnisse über die Wirkungsweise der beteiligten Enzyme das vorstehende Abbauschema (Abb. 7) formulieren. Die Ermittlung der pro Gewichtseinheit des Frischgewebes vorhandenen Enzymaktivitäten und Substratkonzentrationen erlaubt darüber hinaus folgende Schlüsse: Ein Vergleich der in Tabelle 2 zusammen-

Tab. 2

Michaeliskonstanten und Aktivitäten der Glykosamino-glykanohydrolasen der Rinderaorta. Die angegebenen Werte beziehen sich fur Hyaluronat-glykanohydrolase und Chondroitin-4-sulfat-sulfohydrolase auf die bei Umsatz mit Chondroitin-4-sulfat erhaltenen AKtivitäten bei maximaler Reaktionsgeschwindigkeit. Für die $\beta$-Glucuronidase wurden die bei Umsatz mit dem Tetrasaccharid GlcUAGalNAc-4-sulfat-GlcUA-GalNAc-4-sulfat erhaltenen Werte zugrunde gelegt. Die Angaben für die $\beta$-N-Acetylhexosaminidase wurden 1. c. (10), entnommen

\begin{tabular}{|c|c|c|}
\hline & $\begin{array}{l}\text { Enzymaktivität } \\
\text { des } \\
\text { Frischgewebes } \\
\text { mU/g }\end{array}$ & $\underset{\mathrm{mMol} / \text { Liter }}{\mathrm{K}_{\mathrm{m}}}$ \\
\hline $\begin{array}{l}\text { Hyaluronat-glykanohydrolase } \\
\text { Chondroitin-4-sulfat-sulfohydrolase } \\
\beta=\text {-Acetyl-glucosaminidase } \\
\beta \text {-Glucuronidase }\end{array}$ & $\begin{array}{l}0,71 \\
0,30 \\
9,4 \\
8,8\end{array}$ & $\begin{array}{l}1,25 \\
73,5 \\
15,4 \\
11,0\end{array}$ \\
\hline
\end{tabular}

gestellten Aktivitäten der Chondroitin-4-sulfat abbauenden Enzyme zeigt, daß für die Abbausequenz Chondroitin-4-sulfat GlcUA-GalNAc die Chondroitin4-sulfat-sulfohydrolase aufgrund ihrer geringsten spezifischen Aktivität „Schrittmacher-Enzym" ist. Zwar greift die Hyaluronidase auch das nichtdesulfatierte Chondroitin-4-sulfat (allerdings mit geringerer Geschwindigkeit) als Substrat an, der Abbau würde jedoch bei fehlender Chondroitinsulfatase-Aktivität auf der Stufe des GalNAc-4-sulfat-GlcUA-GalNAc-4 sulfat stehenbleiben, da die Hexosaminidase am nichtreduzierenden Ende stehende substituierte N-Acetylhexosaminreste nicht abspaltet (3).

Ob die Schrittmacherfunktion der Chondroitinsulfatase physiologische Bedeutung besitzt, hängt davon $a b, o b$ ihre Kapazität überhaupt in nennenswertem Maße beansprucht wird, $d$. h. letztlich vom Verhältnis zwischen der in $\mathrm{mU}$ aụsgedrückten Enzymaktivität des Arteriengewebes und der Konzentration des angebotenen Substrats im Zustand des „Fließgleichgewichts“ des Stoffwechsels. Für Hyaluronidase und Chondroitin- 
sulfatase sind sowohl Enzymaktivität (mU/g Frischgewebe, s. Tab. 2) als auch die Substratkonzentration (etwa $2 \mathrm{mg}$ Chondroitin-4-sulfat/g Frischgewebe) experimentell bestimmbar. Für Glucuronidase und Hexosaminidase läßt sich dagegen die aktuelle Substratkonzentration lediglich aus dem Umsatz des Chondroitin-4-sulfat in der Arterienwand abschätzen. $\mathrm{Da}$ die Halbwertszeit des Chondroitinsulfat im Arteriengewebe nach Isotopenstudien etwa 10 Tage beträgt (3), errechnet sich die Abbaurate für das Chondroitinsulfat bezogen auf das von der Hyaluronidase als Reaktionsprodukt gebildete und von Glucuronidase und Hexosaminidase als Substrat weiter umgesetzte Tetrasaccharid GlcUA-GalNAc-GlcUA-GalNAc-zu 0,07 $\mu \mathrm{Mol} /$ Min./g Frischgewebe. Die Aktivitäten von Hyaluronidase und Chondroitinsulfatase würden jedoch einen Umsatz von $0,71 \mathrm{bzw} .0,30 \mu \mathrm{Mol} / \mathrm{Min}$./g Frischgewicht, die Aktivitäten von Glucuronidase und Hexosaminidase sogar einen Umsatz von $8,8 \mathrm{bzw}$. 9,4 $\mu \mathrm{Mol} / \mathrm{Min}$./g Frischgewicht zulassen. Es besteht also eine beträcht- liche Differenz zwischen möglicher und tatsächlicher Abbaurate des Chondroitin-4-sulfats, woraus zu folgern ist, daß die „effektive Enzymkonzentration“, d. h. also diejenige Enzymmenge, die mit dem Abbau des Substrates beschäftigt ist, nur einen Bruchteil der totalen Enzymaktivität ausmachen kann. Diese Beobachtung steht im Einklang mit der Tatsache, dảß die Glykosaminoglykanohydrolasen des Arteriengewebes lysosomale Enzyme (12) sind und führt weiterhin zu der Annahme, $\mathrm{da} \beta$ von den in den Lysosomen in aktiver Form lokalisierten Hydrolasen physiologischerweise jeweils nur ein kleiner - nach den vorliegenden Daten einige Prozent betragender - Anteil für den (extrazellulären) Abbau des Chondroitinsulfats zur Verfügung gestellt wird.

Die Deutsche Forschungsgemeinschaft und der Fonds der Chemischen Industrie haben die Arbeit durch Sachbeihilfen und die Gewährung von Ausbildungsstipendien (E. H. und U. St.) in dankenswerter Weise unterstützt.

\section{Literatur}

1. Buddecke E. und M. Schubert, Hoppe-Seyler's Z. physiol. Chem. 325, 189 (1961); BuDDECKE, E., W. KRÖZ und E. LANKA, Hoppe-Seyler's Z. physiol. Chem. 331, 196 (1963). - 2. Hauss, W.H., Verh. Dtsch. Ges. inn. Med. 69, 554 (1963); Hauss, W. H. und G. Junge-HülsIng, Dtsch. med. Wschr. 86, 763 (1961). 3. BudDecke E. und E. Werries, Hoppe-Seyler's Z. physiol. Chem. 340, 257 (1965). - 4. Buddecke, E. und D. Platt, HoppeSeyler's Z. phys. Chem. 343, 61 (1965). - 5. Buddecke, E. und O. Hoefene, Hoppe-Seyler's Z. physiol. Chem. 347, 173 (1966). 6. Buddecke, E., G. ReIch und U. Stein, Hoppe-Seyler's Z. physiol. Chem. 347, 192. (1966). - 7. HrLd E. und E. BudDECKE, Hoppe-Seyler's Z. physiol. Chem. 348, 1047 (1967). - 8. REICH, G. und E. BuDDECKE, Hoppe-Seyler's Z. physiol. Chem. 348, 1616 (1967) - 9. BuddeCKe, E., W. KRöz und W. TitToR, HoppeSeyler's Z. physiol. Chem. 348, 651 (1967). - 10. Werssmann, B., S. Hadkiroannon und J. Tornheim, J. biol. Chemistry 239, 59 (1964). - 11. Green, M. N., K. Tson, R. Bressler und A. M. Seligman, Arch. Biochem. Biophysics 57, 458 (1955); KIRK, J. E., J. Gerontol. 15, 136 (1960). - 12. FurPovic, J. und E. BuDDECKE, Hoppe-Seyler's Z. physiol. Chem. im Druck

Prof. Dr. E. Buddecke 44 Münster (Westf.) Waldeyerstraße 15 